\title{
An Achievable Rate Region for the Two-Way Two-Relay Channel
}

\author{
Jonathan Ponniah and Liang-Liang Xie \\ Department of Electrical and Computer Engineering, \\ University of Waterloo, Canada
}

\begin{abstract}
We propose an achievable rate region for the twoway two-relay channel using decode-and-forward block Markovian coding. A previous attempt to obtain an achievable region for this network encountered a fundamental deadlock problem in which each relay needed to decode before the other to enable mutual assistance. In this paper, the deadlock is resolved by introducing an additional constraint that ensures some relay can decode at least one source before the other relay. The resulting achievable region is an extension of existing results for the twoway single-relay channel.
\end{abstract}

\section{INTRODUCTION}

The two-way relay channel [1] is often cited to explain the benefits of network-coding for the wireless setting. In this channel, two sources communicate to each other with the help of a relay using the network-coding protocol. First, the relay linearly combines (or XORs) the two source messages it has decoded and broadcasts the result. Then the source nodes, knowing what they transmitted in the past, can recover each other's message by decoding the relay transmission and inverting the linear operation. Thus, the flow of information remains uninterrupted despite the common channel between the relay and sources.

More generally, it turns out the idea of network coding can be applied with random binning, a classical technique in multiuser information theory. Under this framework, the following achievable rates for the two-way single-relay channel were obtained in [2],

$$
\begin{aligned}
& R_{1}<I\left(X_{1}, X_{2} ; Y_{3} \mid X_{3}\right) \\
& R_{3}<I\left(X_{2}, X_{3} ; Y_{1} \mid X_{1}\right)
\end{aligned}
$$

and

$$
\begin{aligned}
R_{1} & <I\left(X_{1} ; Y_{2} \mid X_{2}, X_{3}\right) \\
R_{3} & <I\left(X_{3} ; Y_{2} \mid X_{1}, X_{2}\right) \\
R_{1}+R_{3} & <I\left(X_{1}, X_{3} ; Y_{2} \mid X_{2}\right)
\end{aligned}
$$

for any $p\left(x_{1}\right) p\left(x_{2}\right) p\left(x_{3}\right)$, where nodes 1 and 3 are the sources, and node 2 is the relay.

A consequence of the network coding strategy is that the input distribution of each node must be independent, thus precluding the use of beamforming. If we use superposition coding instead, then the input distribution of each node will be correlated, allowing the use of beamforming. Such a scheme for this network was first studied in [3], where, however, it was incorrectly claimed that (1)-(5) are achievable for any $p\left(x_{1} \mid x_{2 f}\right) p\left(x_{3} \mid x_{2 b}\right) p\left(x_{2 f}\right) p\left(x_{2 b}\right)$. The analysis in [3] was limited to the AWGN channel, but erred by not distinguishing the signal power sending superimposed "new" information, from the signal power coherently transmitting "old" information. The correct achievable region based on superposition coding is as follows,

$$
\begin{aligned}
& R_{1}<I\left(X_{1}, X_{2} ; Y_{3} \mid U_{3}, X_{3}\right) \\
& R_{3}<I\left(X_{2}, X_{3} ; Y_{1} \mid U_{1}, X_{1}\right)
\end{aligned}
$$

and

$$
\begin{aligned}
R_{1} & <I\left(X_{1} ; Y_{2} \mid U_{1}, U_{3}, X_{2}, X_{3}\right) \\
R_{3} & <I\left(X_{3} ; Y_{2} \mid U_{1}, U_{3}, X_{1}, X_{2}\right) \\
R_{1}+R_{3} & <I\left(X_{1}, X_{3} ; Y_{2} \mid U_{1}, U_{3}, X_{2}\right)
\end{aligned}
$$

for any $p\left(u_{1}\right) p\left(u_{3}\right) p\left(x_{1} \mid u_{1}\right) p\left(x_{2} \mid u_{1}, u_{3}\right) p\left(x_{3} \mid u_{3}\right)$, where $U_{1}$ and $U_{3}$ are auxiliary random variables.

Depending on the channel parameters, neither of the two achievable regions (1)-(5) and (6)-(10) is always superior than the other. In this paper we will focus on network coding, which is relatively simpler to extend, but the basic strategies can be similarly applied to superposition coding as well.

The achievable rate region in [2] has a simple interpretation; (1) and (2) are cut-set bounds without beamforming, while (3)(5) is the multiple-access region if the relay is to fully decode both sources. Now, an immediate question is whether this interpretation extends to the two-relay case. More specifically, we would like to know if the following rates are achievable in the two-relay setting,

$$
\begin{aligned}
& R_{1}<I\left(X_{1}, X_{2}, X_{3} ; Y_{4} \mid X_{4}\right) \\
& R_{4}<I\left(X_{2}, X_{3}, X_{4} ; Y_{1} \mid X_{1}\right)
\end{aligned}
$$

and

$$
\begin{aligned}
R_{1} & <I\left(X_{1} ; Y_{2} \mid X_{2}, X_{3}, X_{4}\right) \\
R_{4} & <I\left(X_{3}, X_{4} ; Y_{2} \mid X_{1}, X_{2}\right) \\
R_{1}+R_{4} & <I\left(X_{1}, X_{3}, X_{4} ; Y_{2} \mid X_{2}\right)
\end{aligned}
$$

and

$$
\begin{aligned}
R_{1} & <I\left(X_{1}, X_{2} ; Y_{3} \mid X_{3}, X_{4}\right) \\
R_{4} & <I\left(X_{4} ; Y_{3} \mid X_{1}, X_{2}, X_{3}\right) \\
R_{1}+R_{4} & <I\left(X_{1}, X_{2}, X_{4} ; Y_{3} \mid X_{3}\right)
\end{aligned}
$$

where nodes 1 and 4 are the sources, and nodes 2 and 3 are the relays. 
Notice that (11) and (12) correspond to the cut-set bounds. Furthermore, (13)-(15) and (16)-(18) seem reasonable extensions of the multiple access constraints to each relay node. Unfortunately, there is a fundamental difficulty in achieving (11)-(18). To achieve (13)-(15), node 3 needs to decode before node 2 in order to help, but the reverse is also needed for (16)(18). This "deadlock" problem was identified in [4] when a backward decoding scheme was tried for achieving (11)-(18).

In this paper, we resolve the deadlock by adding an additional constraint to (11)-(18) that ensures some relay can decode at least one of the sources before the other relay. Intuitively, this requirement is reasonable; to start the flow of information it is expected that one of the relays must be able to decode a source message first.

There are two ways in which a coding scheme satisfies the additional constraint: in the first case some relay decodes one source before the other relay, and in the second case some relay decodes both sources before the other relay. For each case, we will develop coding schemes that can recover the region defined by (11)-(18). A key ingredient in some of the coding schemes is an offset-encoding strategy developed in [5], that gives more flexibility when combined with slidingwindow decoding. As in [2] the schemes presented in this paper also introduce random binning at the relay nodes.

This paper is organized as follows. In section II, we introduce the two-way two-relay channel model, state the achievable region, and provide an intuitive interpretation of the results. In section III three coding schemes are introduced that collectively achieve the rate region of section II. Section IV concludes the paper.

\section{THE Two-WAY Two-RELAY CHANNEL}

Consider a network of four nodes $1,2,3$, 4, with the inputoutput dynamics modeled by the discrete memoryless channel

$$
\begin{array}{r}
\left(\mathcal{X}_{1} \times \mathcal{X}_{2} \times \mathcal{X}_{3} \times \mathcal{X}_{4}, p\left(y_{1}, y_{2}, y_{3}, y_{4} \mid x_{1}, x_{2}, x_{3}, x_{4}\right)\right. \\
\left.\mathcal{Y}_{1} \times \mathcal{Y}_{2} \times \mathcal{Y}_{3} \times \mathcal{Y}_{4}\right)
\end{array}
$$

That is, at any time $t=1,2, \ldots$, the outputs $y_{1, t}$, $y_{2, t}, y_{3, t}$, and $y_{4, t}$ received by the four nodes respectively only depend on the inputs $x_{1, t}, x_{2, t}, x_{3, t}$, and $x_{4, t}$ transmitted by the four nodes at the same time according to $p\left(y_{1, t}, y_{2, t}, y_{3, t}, y_{4, t} \mid x_{1, t}, x_{2, t}, x_{3, t}, x_{4, t}\right)$.

In the two-way two-relay problem, the source nodes 1 and 4 communicate with each other at rates $R_{1}$ and $R_{4}$ respectively, with the help of the relay nodes 2 and 3 . We are interested in the simultaneously achievable rates $\left(R_{1}, R_{4}\right)$. The standard definitions of codes and achievable rates are omitted, except a special note that during any time $t$, each node $i$ can choose its input $x_{i, t}$ based on the past outputs $\left(y_{i, t-1}, y_{i, t-2}, \ldots, y_{i, 1}\right)$ it has already received.

Theorem 2.1: For the two-way two-relay problem defined above, any rates $\left(R_{1}, R_{4}\right)$ satisfying (11)-(18) are simultaneously achievable, provided that at least one of the following constraints hold:

$$
\begin{aligned}
R_{1} & <I\left(X_{1} ; Y_{2} \mid X_{2}, X_{3}\right) \\
R_{4} & <I\left(X_{4} ; Y_{3} \mid X_{2}, X_{3}\right) \\
R_{1}+R_{4} & <\max \left\{I\left(X_{1}, X_{4} ; Y_{2} \mid X_{2}, X_{3}\right),\right. \\
& \left.I\left(X_{1}, X_{4} ; Y_{3} \mid X_{2}, X_{3}\right)\right\}
\end{aligned}
$$

Observe that by symmetry, it is only necessary to consider two mutually exclusive cases in (19)-(21).

- Case 1 in which (20) holds. In this case, the rate of node 4 is low enough to ensure node 3 can decode the message despite interference from node 1 . More generally, it will be shown that the first case applies when some relay decodes one source before the other relay.

- Case 2 in which the first part of (21) holds but neither (19) nor (20) hold. The throughput is low enough to ensure node 2 can decode without assistance from node 3 but unlike the first case, node 1 does not cause any interference. More generally, it will be shown that the second case applies when some relay decodes both sources before the other relay.

It is emphasized that the rate regions of the first and second cases are different. That is, there exist rate pairs $\left(R_{1}, R_{4}\right)$ that satisfy (19) or (20) but not (21) and vice-versa.

\section{Proof of Theorem 2.1}

Each case is examined separately. In the first case we show there are two coding strategies that together, obtain the rate region defined by (11)-(18) and (20): an offset encoding scheme, and a no-offset encoding scheme. In the second case, we use a multiple-access strategy to recover the rate region defined by (11)-(18) and the first part of (21).

Proof: For any fixed $p\left(x_{1}\right) p\left(x_{2}\right) p\left(x_{3}\right) p\left(x_{4}\right)$, choose

$$
\begin{aligned}
R_{2} \geq & \max \left\{I\left(X_{2} ; Y_{1} \mid X_{1}, X_{3}\right), I\left(X_{2} ; Y_{3} \mid X_{3}, X 4\right)\right. \\
& \left.I\left(X_{2} ; Y_{4} \mid X_{3}, X_{4}\right)\right\} \\
R_{3} \geq & \max \left\{I\left(X_{3} ; Y_{1} \mid X_{1}, X_{2}\right), I\left(X_{3} ; Y_{2} \mid X_{1}, X_{2}\right),\right. \\
& \left.I\left(X_{3} ; Y_{4} \mid X_{4}\right)\right\}
\end{aligned}
$$

We use the Markov block coding argument. Consider $B$ blocks of transmission, each of $n$ transmission slots. For some fixed $K, J \in \mathcal{I}$, a sequence of $B-K$ indices, $w_{1, b} \in$ $\left\{1, \ldots, 2^{n R_{1}}\right\}, b=1,2, \ldots, B-K$ will be sent over from node 1 to node 4 in $n B$ transmission slots, and at the same time, another sequence of $B-J$ indices, $w_{4, b} \in\left\{1, \ldots, 2^{n R_{4}}\right\}$ will be sent over from node 4 to node 1 .

Generation of Codebooks: For each node $i=1, \ldots, 4$ independently generate $2^{n R_{i}} \quad$ i.i.d $n$-sequences $\mathbf{x}_{i}=\left(x_{i, 1}, \ldots, x_{i, n}\right)$ in $\mathcal{X}_{i}^{n}$ according to $p\left(x_{i}\right)$. Index them as $\mathbf{x}_{i}\left(w_{i}\right), w_{i} \in\left\{1,2, \ldots, 2^{n R_{i}}\right\}$.

Random Binning: For each relay node $i=2,3$, generate $2^{n R_{i}}$ bins, indexed by $B_{i}(k)$ with $k=1, \ldots, 2^{n R_{i}}$. Independently throw each index pair $\left(w_{1}, w_{4}\right), w_{1} \in\left\{1,2, \ldots, 2^{n R_{1}}\right\}$, $w_{4} \in\left\{1,2, \ldots, 2^{n R_{4}}\right\}$ into the $2^{n R_{i}}$ bins according to the uniform distribution. Let $k_{i}\left(w_{1}, w_{4}\right)$ be the index of the bin which contains the pair $\left(w_{1}, w_{4}\right)$. 
TABLE I

AN OFFSET ENCODING SCHEME FOR CASE 1

\begin{tabular}{c|l|l|l|l}
\hline \hline Node & Block $b-3$ & Block $b-2$ & Block $b-1$ & Block $b$ \\
\hline 1 & $\mathbf{x}_{1}\left(w_{1, b-3}\right)$ & $\mathbf{x}_{1}\left(w_{1, b-2}\right)$ & $\mathbf{x}_{1}\left(w_{1, b-1}\right)$ & $\mathbf{x}_{1}\left(w_{1, b}\right)$ \\
\hline 2 & $\mathbf{x}_{2}\left(w_{1, b-5}, w_{4, b-5}\right)$ & $\mathbf{x}_{2}\left(w_{1, b-4}, w_{4, b-4}\right)$ & $\mathbf{x}_{2}\left(w_{1, b-3}, w_{4, b-3}\right)$ & $\mathbf{x}_{2}\left(w_{1, b-2}, w_{4, b-2}\right)$ \\
\hline 3 & $\mathbf{x}_{3}\left(w_{1, b-6}, w_{4, b-4}\right)$ & $\mathbf{x}_{3}\left(w_{1, b-5}, w_{4, b-3}\right)$ & $\mathbf{x}_{3}\left(w_{1, b-4}, w_{4, b-2}\right)$ & $\mathbf{x}_{3}\left(w_{1, b-3}, w_{4, b-1}\right)$ \\
\hline 4 & $\mathbf{x}_{4}\left(w_{4, b-3}\right)$ & $\mathbf{x}_{4}\left(w_{4, b-2}\right)$ & $\mathbf{x}_{4}\left(w_{4, b-1}\right)$ & $\mathbf{x}_{4}\left(w_{4, b}\right)$ \\
\hline
\end{tabular}

\section{A. Case 1: An Offset Encoding Scheme}

Encoding: We use an offset encoding scheme to obtain an achievable rate region consistent with (20). The encoding scheme is depicted in table I. It is assumed that a message pair transmitted in block $b$ was decoded at the end of block $b-1$. An interesting feature of this coding scheme is that node 2 decodes the message from node 1 based on the signals received over two blocks (not one block).

Decoding:

1) At the end of each block $b=3, \ldots, B$, node 1 determines the unique index $\hat{w}_{4, b-2}$ that satisfies the joint typicality checks:

$$
\begin{aligned}
& \left(\mathbf{X}_{1, b-2}, \mathbf{x}_{2}\left(k_{2}\left(w_{1, b-4}, w_{4, b-4}\right)\right), \mathbf{x}_{3}\left(k_{3}\left(w_{1, b-5}, w_{4, b-3}\right)\right),\right. \\
& \left.\quad \mathbf{x}_{4}\left(\hat{w}_{4, b-2}\right), \mathbf{Y}_{1, b-2}\right) \in A_{\epsilon}^{(n)}\left(X_{1}, X_{2}, X_{3}, X_{4}, Y_{1}\right) \\
& \left(\mathbf{X}_{1, b-1}, \mathbf{x}_{2}\left(k_{2}\left(w_{1, b-3}, w_{4, b-3}\right)\right), \mathbf{x}_{3}\left(k_{3}\left(w_{1, b-4}, \hat{w}_{4, b-2}\right)\right),\right. \\
& \left.\quad \mathbf{Y}_{1, b-1}\right) \in A_{\epsilon}^{(n)}\left(X_{1}, X_{2}, X_{3}, Y_{1}\right) \\
& \left(\mathbf{X}_{1, b}, \mathbf{x}_{2}\left(k_{2}\left(w_{1, b-2}, \hat{w}_{4, b-2}\right)\right), \mathbf{Y}_{1, b}\right) \in A_{\epsilon}^{(n)}\left(X_{1}, X_{2}, Y_{1}\right)
\end{aligned}
$$

2) At the end of each block $b=2, \ldots, B$, node 2 determines the unique index pair $\left(\hat{w}_{1, b-1}, \hat{w}_{4, b-1}\right)$ that satisfies the joint typicality checks:

$$
\begin{aligned}
& \left(\mathbf{x}_{1}\left(\hat{w}_{1, b-1}\right), \mathbf{X}_{2, b-1}, \mathbf{x}_{3}\left(k_{3}\left(w_{1, b-4}, w_{4, b-2}\right)\right), \mathbf{x}_{4}\left(\hat{w}_{4, b-1}\right),\right. \\
& \left.\mathbf{Y}_{2, b-1}\right) \in A_{\epsilon}^{(n)}\left(X_{1}, X_{2}, X_{3}, X_{4}, Y_{2}\right) \\
& \left(\mathbf{X}_{2, b}, \mathbf{x}_{3}\left(k_{3}\left(w_{1, b-3}, \hat{w}_{4, b-1}\right)\right), \mathbf{Y}_{2, b}\right) \in A_{\epsilon}^{(n)}\left(X_{2}, X_{3}, Y_{2}\right)
\end{aligned}
$$

3 ) At the end of each block $b=1, \ldots, B$, node 3 determines the unique index pair $\left(\hat{w}_{1, b-2}, \hat{w}_{4, b}\right)$ that satisfies the joint typicality checks:

$$
\begin{aligned}
& \left(\mathbf{x}_{1}\left(\hat{w}_{1, b-2}\right), \mathbf{x}_{2}\left(k_{2}\left(w_{1, b-4}, w_{4, b-4}\right)\right), \mathbf{X}_{3, b-2}, \mathbf{x}_{4}\left(w_{4, b-2}\right),\right. \\
& \left.\quad \mathbf{Y}_{3, b-2}\right) \in A_{\epsilon}^{(n)}\left(X_{1}, X_{2}, X_{3}, X_{4}, Y_{3}\right) \\
& \left(\mathbf{x}_{2}\left(k_{2}\left(\hat{w}_{1, b-2}, w_{4, b-2}\right)\right), \mathbf{X}_{3, b}, \mathbf{x}_{4}\left(\hat{w}_{4, b}\right), \mathbf{Y}_{3, b}\right) \\
& \quad \in A_{\epsilon}^{(n)}\left(X_{2}, X_{3}, X_{4}, Y_{3}\right)
\end{aligned}
$$

4) At the end of each block $b=4, \ldots, B$, node 4 determines the unique index $\hat{w}_{1, b-3}$ that satisfies the joint typicality checks:

$$
\begin{aligned}
& \left(\mathbf{x}_{1}\left(\hat{w}_{1, b-3}\right), \mathbf{x}_{2}\left(k_{2}\left(w_{1, b-5}, w_{4, b-5}\right)\right),\right. \\
& \left.\quad \mathbf{x}_{3}\left(k_{3}\left(w_{1, b-6}, w_{4, b-4}\right)\right), \mathbf{X}_{4, b-3}, \mathbf{Y}_{4, b-3}\right) \\
& \quad \in A_{\epsilon}^{(n)}\left(X_{1}, X_{2}, X_{3}, X_{4}, Y_{4}\right) \\
& \left(\mathbf{x}_{2}\left(k_{2}\left(\hat{w}_{1, b-3}, w_{4, b-3}\right)\right), \mathbf{x}_{3}\left(k_{3}\left(w_{1, b-4}, w_{4, b-2}\right)\right),\right. \\
& \left.\quad \mathbf{X}_{4, b-1}, \mathbf{Y}_{4, b-1}\right) \in A_{\epsilon}^{(n)}\left(X_{2}, X_{3}, X_{4}, Y_{4}\right) \\
& \left(\mathbf{x}_{3}\left(k_{3}\left(\hat{w}_{1, b-3}, w_{4, b-1}\right)\right), \mathbf{X}_{4, b}, \mathbf{Y}_{4, b}\right) \in A_{\epsilon}^{(n)}\left(X_{3}, X_{4}, Y_{4}\right)
\end{aligned}
$$

\section{Analysis of Probability of Error:}

1) In block $b$, node 1 can decode $w_{4, b-2}$ with arbitrarily small probability of error if

$$
\begin{aligned}
R_{4}< & I\left(X_{4} ; Y_{1} \mid X_{1}, X_{2}, X_{3}\right)+I\left(X_{3} ; Y_{1} \mid X_{1}, X_{2}\right) \\
& +I\left(X_{2} ; Y_{1} \mid X_{1}\right)
\end{aligned}
$$

where the three mutual informations follow from the three typicality checks respectively and their combination leads to (12).

2) In block $b$, node 2 can decode the pair $\left(w_{1, b-1}, w_{4, b-1}\right)$ with arbitrarily small probability of error if

$$
\begin{aligned}
R_{1}< & I\left(X_{1} ; Y_{2} \mid X_{2}, X_{3}, X_{4}\right) \\
R_{4}< & I\left(X_{4} ; Y_{2} \mid X_{1}, X_{2}, X_{3}\right) \\
& +I\left(X_{3} ; Y_{2} \mid X_{2}\right) \\
R_{1}+R_{4}< & I\left(X_{1}, X_{4} ; Y_{2} \mid X_{2}, X_{3}\right)+I\left(X_{3} ; Y_{2} \mid X_{2}\right) \\
= & I\left(X_{1}, X_{3}, X_{4} ; Y_{2} \mid X_{2}\right)
\end{aligned}
$$

where each inequality corresponds to one of the three ways a message pair can be decoded incorrectly.

3 ) In block $b$, node 3 can decode the pair $\left(w_{1, b-2}, w_{4, b}\right)$ with arbitrarily small probability of error if

$$
\begin{aligned}
R_{1} & <I\left(X_{1} ; Y_{3} \mid X_{2}, X_{3}, X_{4}\right)+I\left(X_{2} ; Y_{3} \mid X_{3}, X_{4}\right) \\
& =I\left(X_{1}, X_{2} ; Y_{3} \mid X_{3}, X_{4}\right) \\
R_{4} & <I\left(X_{4} ; Y_{3} \mid X_{2}, X_{3}\right) \\
R_{1}+R_{4} & <I\left(X_{1} ; Y_{3} \mid X_{2}, X_{3}, X_{4}\right)+I\left(X_{2}, X_{4} ; Y_{3} \mid X_{3}\right) \\
& =I\left(X_{1}, X_{2}, X_{4} ; Y_{3} \mid X_{3}\right)
\end{aligned}
$$

4) In block $b$, node 4 can decode the message $w_{1, b-3}$ with arbitrarily small probability of error if

$$
\begin{aligned}
R_{1}< & I\left(X_{1} ; Y_{4} \mid X_{2}, X_{3}, X_{4}\right)+I\left(X_{2} ; Y_{4} \mid X_{3}, X_{4}\right) \\
& +I\left(X_{3} ; Y_{4} \mid X_{4}\right)
\end{aligned}
$$

where the three mutual informations lead to (11).

\section{B. Case 1: A No-offset Encoding Scheme}

Encoding: We use a no-offset encoding scheme to obtain an achievable region consistent with (20). The encoding scheme is depicted in table II.

\section{Decoding:}

1) At the end of each block $b=3, \ldots, B$, node 1 determines the unique index $\hat{w}_{4, b-2}$ that satisfies the joint 
typicality checks:

TABLE II

$$
\begin{aligned}
& \left(\mathbf{X}_{1, b-2}, \mathbf{x}_{2}\left(k_{2}\left(w_{1, b-3}, w_{4, b-4}\right)\right), \mathbf{x}_{3}\left(k_{3}\left(w_{1, b-4}, w_{4, b-3}\right)\right),\right. \\
& \left.\quad \mathbf{x}_{4}\left(\hat{w}_{4, b-2}\right), \mathbf{Y}_{1, b-2}\right) \in A_{\epsilon}^{(n)}\left(X_{1}, X_{2}, X_{3}, X_{4}, Y_{1}\right) \\
& \left(\mathbf{X}_{1, b-1}, \mathbf{x}_{2}\left(k_{2}\left(w_{1, b-2}, w_{4, b-3}\right)\right), \mathbf{x}_{3}\left(k_{3}\left(w_{1, b-3}, \hat{w}_{4, b-2}\right)\right),\right. \\
& \left.\quad \mathbf{Y}_{1, b-1}\right) \in A_{\epsilon}^{(n)}\left(X_{1}, X_{2}, X_{3}, Y_{1}\right) \\
& \left(\mathbf{X}_{1, b}, \mathbf{x}_{2}\left(k_{2}\left(w_{1, b-1}, \hat{w}_{4, b-2}\right)\right), \mathbf{Y}_{1, b}\right) \in A_{\epsilon}^{(n)}\left(X_{1}, X_{2}, Y_{1}\right)
\end{aligned}
$$

\begin{tabular}{c|l|l|l}
\hline \hline Node & Block $b-2$ & Block $b-1$ & Block $b$ \\
\hline 1 & $\mathbf{x}_{1}\left(w_{1, b-2}\right)$ & $\mathbf{x}_{1}\left(w_{1, b-1}\right)$ & $\mathbf{x}_{1}\left(w_{1, b}\right)$ \\
\hline 2 & $\mathbf{x}_{2}\left(w_{1, b-3}, w_{4, b-4}\right)$ & $\mathbf{x}_{2}\left(w_{1, b-2}, w_{4, b-3}\right)$ & $\mathbf{x}_{2}\left(w_{1, b-1}, w_{4, b}-2\right)$ \\
\hline 3 & $\mathbf{x}_{3}\left(w_{1, b-4}, w_{4, b-3}\right)$ & $\mathbf{x}_{3}\left(w_{1, b-3}, w_{4, b-2}\right)$ & $\mathbf{x}_{3}\left(w_{1, b-2}, w_{4, b}-1\right)$ \\
\hline 4 & $\mathbf{x}_{4}\left(w_{4, b-2}\right)$ & $\mathbf{x}_{4}\left(w_{4, b-1}\right)$ & $\mathbf{x}_{4}\left(w_{4, b}\right)$ \\
\hline
\end{tabular}

2) At the end of each block $b=1, \ldots, B$, node 2 determines the unique index pair $\left(\hat{w}_{1, b}, \hat{w}_{4, b-1}\right)$ that satisfies the joint typicality checks:

$$
\begin{aligned}
& \left(\mathbf{x}_{1}\left(w_{1, b-1}\right), \mathbf{X}_{2, b-1}, \mathbf{x}_{3}\left(k_{3}\left(w_{1, b-3}, w_{4, b-2}\right)\right), \mathbf{x}_{4}\left(\hat{w}_{4, b-1}\right),\right. \\
& \left.\quad \mathbf{Y}_{2, b-1}\right) \in A_{\epsilon}^{(n)}\left(X_{1}, X_{2}, X_{3}, X_{4}, Y_{2}\right) \\
& \left(\mathbf{x}_{1}\left(\hat{w}_{1, b}\right), \mathbf{X}_{2, b}, \mathbf{x}_{3}\left(k_{3}\left(w_{1, b-2}, \hat{w}_{4, b-1}\right)\right), \mathbf{Y}_{2, b}\right) \\
& \quad \in A_{\epsilon}^{(n)}\left(X_{1}, X_{2}, X_{3}, Y_{2}\right)
\end{aligned}
$$

3) At the end of each block $b=1, \ldots, B$, node 3 determines the unique index pair $\left(\hat{w}_{1, b-1}, \hat{w}_{4, b}\right)$ that satisfies the joint typicality checks:

$$
\begin{aligned}
& \left(\mathbf{x}_{1}\left(\hat{w}_{1, b-1}\right), \mathbf{x}_{2}\left(k_{2}\left(w_{1, b-2}, w_{4, b-3}\right)\right), \mathbf{X}_{3, b-1}, \mathbf{x}_{4}\left(w_{4, b-1}\right),\right. \\
& \left.\quad \mathbf{Y}_{3, b-1}\right) \in A_{\epsilon}^{(n)}\left(X_{1}, X_{2}, X_{3}, X_{4}, Y_{3}\right) \\
& \left(\mathbf{x}_{2}\left(k_{2}\left(\hat{w}_{1, b-1}, w_{4, b-2}\right)\right), \mathbf{X}_{3, b}, \mathbf{x}_{4}\left(\hat{w}_{4, b}\right), \mathbf{Y}_{3, b}\right) \\
& \quad \in A_{\epsilon}^{(n)}\left(X_{2}, X_{3}, X_{4}, Y_{3}\right)
\end{aligned}
$$

4) At the end of each block $b=3, \ldots, B$, node 4 determines the unique index $\hat{w}_{1, b-2}$ that satisfies the joint typicality checks:

$$
\begin{aligned}
& \left(\mathbf{x}_{1}\left(\hat{w}_{1, b-2}\right), \mathbf{x}_{2}\left(k_{2}\left(w_{1, b-3}, w_{4, b-4}\right)\right),\right. \\
& \left.\quad \mathbf{x}_{3}\left(k_{3}\left(w_{1, b-4}, w_{4, b-3}\right)\right), \mathbf{X}_{4, b-2}, \mathbf{Y}_{4, b-2}\right) \\
& \quad \in A_{\epsilon}^{(n)}\left(X_{1}, X_{2}, X_{3}, X_{4}, Y_{4}\right) \\
& \left(\mathbf{x}_{2}\left(k_{2}\left(\hat{w}_{1, b-2}, w_{4, b-3}\right)\right), \mathbf{x}_{3}\left(k_{3}\left(w_{1, b-3}, w_{4, b-2}\right)\right),\right. \\
& \left.\quad \mathbf{X}_{4, b-1}, \mathbf{Y}_{4, b-1}\right) \in A_{\epsilon}^{(n)}\left(X_{2}, X_{3}, X_{4}, Y_{4}\right) \\
& \left(\mathbf{x}_{3}\left(k_{3}\left(\hat{w}_{1, b-2}, w_{4, b-1}\right)\right), \mathbf{X}_{4, b}, \mathbf{Y}_{4, b}\right) \in A_{\epsilon}^{(n)}\left(X_{3}, X_{4}, Y_{4}\right)
\end{aligned}
$$

Analysis of Probability of Error:

1) In block $b$, node 1 can decode $w_{4, b-2}$ with arbitrarily small probability of error if

$$
\begin{aligned}
R_{4}< & I\left(X_{4} ; Y_{1} \mid X_{1}, X_{2}, X_{3}\right)+I\left(X_{3} ; Y_{1} \mid X_{1}, X_{2}\right) \\
& +I\left(X_{2} ; Y_{1} \mid X_{1}\right)
\end{aligned}
$$

where the combination of the three mutual informations leads to (12).

2) In block $b$, node 2 can decode the pair $\left(w_{1, b}, w_{4, b-1}\right)$ with arbitrarily small probability of error if

$$
\begin{aligned}
R_{1}< & I\left(X_{1} ; Y_{2} \mid X_{2}, X_{3}\right) \\
R_{4}< & I\left(X_{4} ; Y_{2} \mid X_{1}, X_{2}, X_{3}\right) \\
& +I\left(X_{3} ; Y_{2} \mid X_{1}, X_{2}\right) \\
= & I\left(X_{3}, X_{4} ; Y_{2} \mid X_{1}, X_{2}\right) \\
R_{1}+R_{4}< & I\left(X_{4} ; Y_{2} \mid X_{1}, X_{2}, X_{3}\right) \\
& +I\left(X_{1}, X_{3} ; Y_{2} \mid X_{2}\right) \\
= & I\left(X_{1}, X_{3}, X_{4} ; Y_{2} \mid X_{2}\right)
\end{aligned}
$$

3) In block $b$, node 3 can decode the pair $\left(w_{1, b-1}, w_{4, b}\right)$ with arbitrarily small probability of error if

$$
\begin{aligned}
R_{1}< & I\left(X_{1} ; Y_{3} \mid X_{2}, X_{3}, X_{4}\right) \\
& +I\left(X_{2} ; Y_{3} \mid X_{3}, X_{4}\right) \\
= & I\left(X_{1}, X_{2} ; Y_{3} \mid X_{3}, X_{4}\right) \\
R_{4}< & I\left(X_{4} ; Y_{3} \mid X_{2}, X_{3}\right) \\
R_{1}+R_{4}< & I\left(X_{1} ; Y_{3} \mid X_{2}, X_{3}, X_{4}\right)+I\left(X_{2}, X_{4} ; Y_{3} \mid X_{3}\right) \\
= & I\left(X_{1}, X_{2}, X_{4} ; Y_{3} \mid X_{3}\right)
\end{aligned}
$$

4) In block $b$, node 4 can decode the message $w_{1, b-2}$ with arbitrarily small probability of error if

$$
\begin{aligned}
R_{1}< & I\left(X_{1} ; Y_{4} \mid X_{2}, X_{3}, X_{4}\right)+I\left(X_{2} ; Y_{4} \mid X_{3}, X_{4}\right) \\
& +I\left(X_{3} ; Y_{4} \mid X_{4}\right)
\end{aligned}
$$

where the combination of the three mutual informations leads to (11).

The combined rate region obtained from the offset and nooffset coding schemes is given by (11)-(18) and (20). This statement is verified by observing that (23) and (22) imply (15). In other words, we have shown that if (20) is true, there are two coding schemes that together recover the region defined by (11)-(18). In both schemes, node 3 decodes $w_{4, b}$ before node 2 , which is consistent with the practical interpretation of case 1 .

\section{Case 2: A Multiple-access Scheme}

Encoding: We present a scheme that obtains an achievable rate region consistent with the first part of (21) given that neither (19) nor (20) holds. The encoding scheme is depicted in table III.

\section{Decoding:}

1) At the end of each block $b=3, \ldots, B$, node 1 determines the unique index $\hat{w}_{4, b-2}$ that satisfies the joint typicality checks:

$$
\begin{aligned}
& \left(\mathbf{X}_{1, b-2}, \mathbf{x}_{2}\left(k_{2}\left(w_{1, b-3}, w_{4, b-3}\right)\right), \mathbf{x}_{3}\left(k_{3}\left(w_{1, b-4}, w_{4, b-4}\right)\right),\right. \\
& \left.\quad \mathbf{x}_{4}\left(\hat{w}_{4, b-2}\right), \mathbf{Y}_{1, b-2}\right) \in A_{\epsilon}^{(n)}\left(X_{1}, X_{2}, X_{3}, X_{4}, Y_{1}\right) \\
& \left(\mathbf{X}_{1, b-1}, \mathbf{x}_{2}\left(k_{2}\left(w_{1, b-2}, \hat{w}_{4, b-2}\right)\right), \mathbf{x}_{3}\left(k_{3}\left(w_{1, b-3}, w_{4, b-3}\right)\right),\right. \\
& \left.\quad \mathbf{Y}_{1, b-1}\right) \in A_{\epsilon}^{(n)}\left(X_{1}, X_{2}, X_{3}, Y_{1}\right) \\
& \left(\mathbf{X}_{1, b}, \mathbf{x}_{3}\left(k_{3}\left(w_{1, b-2}, \hat{w}_{4, b-2}\right)\right), \mathbf{Y}_{1, b}\right) \in A_{\epsilon}^{(n)}\left(X_{1}, X_{3}, Y_{1}\right)
\end{aligned}
$$

2) At the end of each block $b=1, \ldots, B$, node 2 determines the unique index pair $\left(\hat{w}_{1, b}, \hat{w}_{4, b}\right)$ that satisfies the 
joint typicality check:

$$
\begin{aligned}
& \left(\mathbf{x}_{1}\left(\hat{w}_{1, b}\right), \mathbf{X}_{2, b}, \mathbf{x}_{3}\left(k_{3}\left(w_{1, b-2}, w_{4, b-2}\right)\right), \mathbf{x}_{4}\left(\hat{w}_{4, b}\right), \mathbf{Y}_{2, b}\right) \\
& \quad \in A_{\epsilon}^{(n)}\left(X_{1}, X_{2}, X_{3}, X_{4}, Y_{2}\right)
\end{aligned}
$$

3) At the end of each block $b=2, \ldots, B$, node 3 determines the unique index pair $\left(\hat{w}_{1, b-1}, \hat{w}_{4, b-1}\right)$ that satisfies the joint typicality checks:

$$
\begin{aligned}
& \left(\mathbf{x}_{1}\left(\hat{w}_{1, b-1}\right), \mathbf{x}_{2}\left(k_{2}\left(w_{1, b-2}, w_{4, b-2}\right)\right), \mathbf{X}_{3, b-1}, \mathbf{x}_{4}\left(\hat{w}_{4, b-1}\right),\right. \\
& \left.\mathbf{Y}_{3, b-1}\right) \in A_{\epsilon}^{(n)}\left(X_{1}, X_{2}, X_{3}, X_{4}, Y_{3}\right) \\
& \left(\mathbf{x}_{2}\left(k_{2}\left(\hat{w}_{1, b-1}, \hat{w}_{4, b-1}\right)\right), \mathbf{X}_{3, b}, \mathbf{Y}_{3, b}\right) \in A_{\epsilon}^{(n)}\left(X_{2}, X_{3}, Y_{3}\right)
\end{aligned}
$$

4) At the end of each block $b=3, \ldots, B$, node 4 determines the unique index $\hat{w}_{1, b-2}$ that satisfies the joint typicality checks:

$$
\begin{aligned}
& \left(\mathbf{x}_{1}\left(\hat{w}_{1, b-2}\right), \mathbf{x}_{2}\left(k_{2}\left(w_{1, b-3}, w_{4, b-3}\right)\right),\right. \\
& \quad \mathbf{x}_{3}\left(k_{3}\left(w_{1, b-4}, w_{4, b-4}\right)\right), \mathbf{X}_{4, b-2}, \\
& \left.\quad \mathbf{Y}_{4, b-2}\right) \in A_{\epsilon}^{(n)}\left(X_{1}, X_{2}, X_{3}, X_{4}, Y_{4}\right) \\
& \left(\mathbf{x}_{2}\left(k_{2}\left(\hat{w}_{1, b-2}, w_{4, b-2}\right)\right), \mathbf{x}_{3}\left(k_{3}\left(w_{1, b-3}, w_{4, b-3}\right)\right),\right. \\
& \left.\quad \mathbf{X}_{4, b-1}, \mathbf{Y}_{4, b-1}\right) \in A_{\epsilon}^{(n)}\left(X_{2}, X_{3}, X_{4}, Y_{4}\right) \\
& \left(\mathbf{x}_{3}\left(k_{3}\left(\hat{w}_{1, b-2}, w_{4, b-2}\right)\right), \mathbf{X}_{4, b}, \mathbf{Y}_{4, b}\right) \in A_{\epsilon}^{(n)}\left(X_{3}, X_{4}, Y_{4}\right)
\end{aligned}
$$

Analysis of Probability of Error:

1) In block $b$, node 1 can decode $w_{4, b-2}$ with arbitrarily small probability of error if

$$
\begin{aligned}
R_{4}< & I\left(X_{4} ; Y_{1} \mid X_{1}, X_{2}, X_{3}\right)+I\left(X_{2} ; Y_{1} \mid X_{1}, X_{3}\right) \\
& +I\left(X_{3} ; Y_{1} \mid X_{1}\right)
\end{aligned}
$$

where the combination of the three mutual informations leads to (12).

2) In block $b$, node 2 can decode the pair $\left(w_{1, b}, w_{4, b}\right)$ with arbitrarily small probability of error if

$$
\begin{aligned}
R_{1} & <I\left(X_{1} ; Y_{2} \mid X_{2}, X_{3}, X_{4}\right) \\
R_{4} & <I\left(X_{4} ; Y_{2} \mid X_{1}, X_{2}, X_{3}\right) \\
R_{1}+R_{4} & <I\left(X_{1}, X_{4} ; Y_{2} \mid X_{2}, X_{3}\right)
\end{aligned}
$$

3 ) In block $b$, node 3 can decode the pair $\left(w_{1, b-1}, w_{4, b-1}\right)$ with arbitrarily small probability of error if

$$
\begin{aligned}
R_{1}< & I\left(X_{1} ; Y_{3} \mid X_{2}, X_{3}, X_{4}\right) \\
& +I\left(X_{2} ; Y_{3} \mid X_{3}\right) \\
R_{4}< & I\left(X_{4} ; Y_{3} \mid X_{1}, X_{2}, X_{3}\right) \\
& +I\left(X_{2} ; Y_{3} \mid X_{3}\right) \\
R_{1}+R_{4}< & I\left(X_{1}, X_{4} ; Y_{3} \mid X_{2}, X_{3}\right) \\
& +I\left(X_{2} ; Y_{3} \mid X_{3}\right) \\
= & I\left(X_{1}, X_{2}, X_{4} ; Y_{3} \mid X_{3}\right)
\end{aligned}
$$

4) In block $b$, node 4 can decode the message $w_{1, b-2}$ with arbitrarily small probability of error if

$$
\begin{aligned}
R_{1}< & I\left(X_{1} ; Y_{4} \mid X_{2}, X_{3}, X_{4}\right)+I\left(X_{2} ; Y_{4} \mid X_{3}, X_{4}\right) \\
& +I\left(X_{3} ; Y_{4} \mid X_{4}\right)
\end{aligned}
$$

TABLE III

A MULTIPLE-ACCESS ENCODING SCHEME FOR CASE 2

\begin{tabular}{c|l|l|l}
\hline \hline Node & Block $b-2$ & Block $b-1$ & Block $b$ \\
\hline 1 & $\mathbf{x}_{1}\left(w_{1, b-2}\right)$ & $\mathbf{x}_{1}\left(w_{1, b-1}\right)$ & $\mathbf{x}_{1}\left(w_{1, b}\right)$ \\
\hline 2 & $\mathbf{x}_{2}\left(w_{1, b-3}, w_{4, b-3}\right)$ & $\mathbf{x}_{2}\left(w_{1, b-2}, w_{4, b-2}\right)$ & $\mathbf{x}_{2}\left(w_{1, b-1}, w_{4, b}-1\right)$ \\
\hline 3 & $\mathbf{x}_{3}\left(w_{1, b-4}, w_{4, b-4}\right)$ & $\mathbf{x}_{3}\left(w_{1, b-3}, w_{4, b-3}\right)$ & $\mathbf{x}_{3}\left(w_{1, b-2}, w_{4, b}-2\right)$ \\
\hline 4 & $\mathbf{x}_{4}\left(w_{4, b-2}\right)$ & $\mathbf{x}_{4}\left(w_{4, b-1}\right)$ & $\mathbf{x}_{4}\left(w_{4, b}\right)$ \\
\hline
\end{tabular}

where the combination of the three mutual informations leads to (11).

Observe that node 2 decodes both source messages $\left(w_{1, b}, w_{4, b}\right)$ before node 3 , which is consistent with the practical interpretation of case 2 . Now from the definition of this case we know that $\left(R_{1}, R_{4}\right)$ satisfies the following inequalities:

$$
\begin{aligned}
R_{1} & \geq I\left(X_{1} ; Y_{2} \mid X_{2}, X_{3}\right) \\
R_{4} & \geq I\left(X_{4} ; Y_{3} \mid X_{2}, X_{3}\right) \\
R_{1}+R_{4} & <I\left(X_{1}, X_{4} ; Y_{2} \mid X_{2}, X_{3}\right)
\end{aligned}
$$

The constraints (30)-(32) are sufficient to obtain the region defined by (11)-(18) using the third coding scheme. This statement is proved by observing that (30) and (32) imply (25), (18) and (31) imply (27), and (17) implies (28). Furthermore, if either (30) or (31) does not hold, then the rate pair will be included in case 1 .

Therefore it follows from symmetry and the achievable rate regions in case 1 and 2, that one of the constraints (19)-(21) is sufficient to obtain the region defined by (11)-(18). Thus theorem 2.1 is proved.

\section{CONCLUding REMARKS}

We have obtained an achievable rate region for the two-way two-relay channel. Moreover, the deadlock problem caused by (11)-(18) was resolved by an additional constraint (19)-(21). This constraint ensures that some relay will be able to decode at least one source before the other relay.

\section{ACKNOWLEDGEMENTS}

The authors would like to thank one of the reviewers for suggesting the better name "deadlock".

\section{REFERENCES}

[1] Y. Wu, P. A. Chou, and S.-Y. Kung, "Information exchange in wireless networks with network coding and physical-layer broadcast," in Proceedings of the 2005 Conf. on Information Sciences and Systems, (The Johns Hopkins University), March 2005.

[2] L.-L. Xie, "Network Coding and Random Binning for Multi-User Channels," Proc. IEEE Canadian Workshop on Information Theory (CWIT), Edmonton, Canada, June 2007.

[3] B. Rankov and A. Wittneben, "Achievable Rate Regions for the Twoway Relay Channel," Proc. IEEE Int. Symposium on Information Theory (ISIT), Seattle, USA, July 2006.

[4] L.-L. Xie and P. R. Kumar, " Multi-source, multi-destination, multi-relay wireless networks". IEEE Trans. Inf. Theory, vol. 53, no. 10, pp. 35863895, October 2007.

[5] L. Sankar, G. Kramer, and N.B. Mandayam, "Offset encoding for multiaccess relay channels," IEEE Trans. Inf. Theory, vol. 53, no. 10, pp. 3814-3821, Oct. 2007. 